\title{
ICES IN STAR FORMING REGIONS
}

\author{
A.G.G.M. TIELENS \\ Nasa Ames Research Center \\ MS 245-3, Moffett Field, CA 94035-1000, USA \\ AND \\ D.C.B. WHITTET \\ Department of Physics, Applied Physics and Astronomy \\ Rensselaer Polytechnic Institute, Troy, NY 12180, USA
}

\begin{abstract}
IR spectra of sources associated with molecular cloud material show a variety of absorption features attributed to simple molecules, such as $\mathrm{H}_{2} \mathrm{O}, \mathrm{CO}, \mathrm{CH}_{3} \mathrm{OH}, \mathrm{CO}_{2}, \mathrm{CH}_{4}$, and OCS in icy grain mantles. These identifications are reviewed. These molecules are formed through accretion and reaction of gas phase species on grain surfaces. The high abundance of $\mathrm{CH}_{3} \mathrm{OH}$ and $\mathrm{CO}_{2}$ point towards the importance of hydrogenation and oxidation reactions in this process. Observations also show that thermal outgassing is of great importance for the composition of interstellar ice mantles. Both these processes are discussed in some detail.
\end{abstract}

\section{Introduction}

Interstellar ice is an important component of interstellar dust inside molecular clouds. Observations show that typically $10 \%$ of the elemental oxygen is locked up in solid $\mathrm{H}_{2} \mathrm{O}$, comparable to the gas phase abundance of $\mathrm{CO}$. These ices are formed by accretion and reaction on preexisting grain cores and their composition allows us to probe grain surface chemistry directly. It is commonly thought that gaseous $\mathrm{H}_{2}$ is formed through reactions on grain surfaces (Hollenbach \& Salpeter 1971). Other gaseous molecules may be formed that way as well. In particular, the composition of Hot Cores around luminous protostars may reflect the evaporation of ice mantles formed during the preceding dark cloud phase (Walmsley 1989). It has also been argued that processing of these ice mantles by FUV radiation 


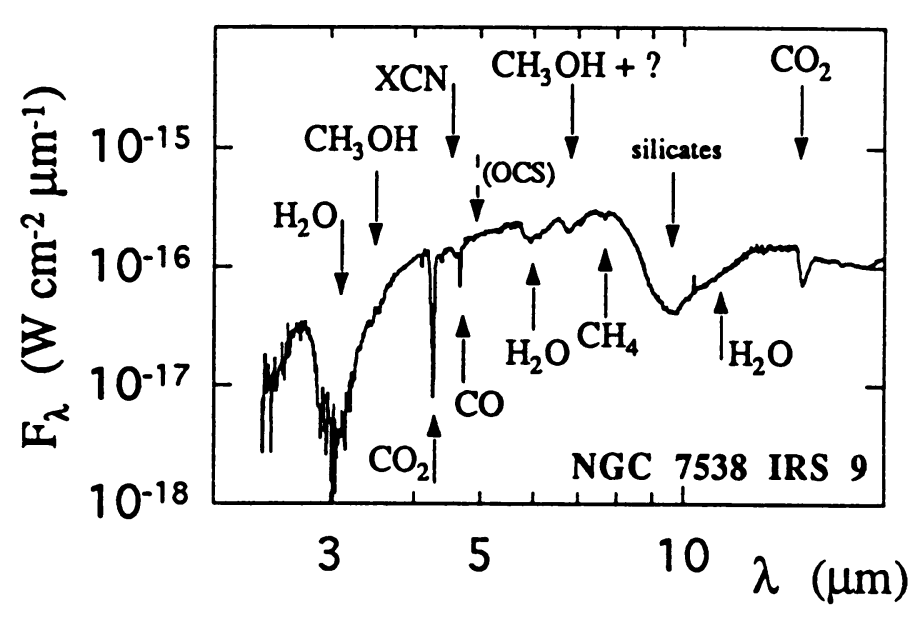

Figure 1. The 2-20 $\mu \mathrm{m}$ spectrum of NGC 7538 IRS 9 obtained with the SWS on ISO at a resolution of 250 (Whittet et al. 1996). Except for the $10 \mu \mathrm{m}$ silicate band, all features are due to simple molecules in ice mantles. Identifications are discussed in the text.

can lead to the formation of organic refractory grain mantles, which might be more resistent against various destruction processes in the ISM and, hence, might be an important dust component in the diffuse ISM (Greenberg 1982). The composition and chemistry of interstellar ices has been reviewed by Whittet (1993), Tielens (1989), and Tielens \& Allamandola $(1987 \mathrm{a}, \mathrm{b})$. First, we review the composition of interstellar ice, emphasizing new results obtained by the SWS on ISO ( $(2)$. Then, we discuss grain surface chemistry, focussing on hydrogenation of $\mathrm{CO}$ into $\mathrm{H}_{2} \mathrm{CO}$ and $\mathrm{CH}_{3} \mathrm{OH}$ (§3), and the origin of the multiple ice mantle components (§4). A summary is presented in $\S 5$.

\section{IR spectroscopy of interstellar ices}

IR spectra of objects embedded in or located behind molecular clouds show a variety of solid state absorption features due to simple species: $\mathrm{H}_{2} \mathrm{O}$, $\mathrm{CH}_{3} \mathrm{OH}, \mathrm{CO}, \mathrm{CO}_{2}, \mathrm{OCS}, \mathrm{XCN}$, and $\mathrm{CH}_{4}$. Figure 1 shows as an example the ISO SWS 2.6-16 $\mu \mathrm{m}$ spectrum of NGC 7538 IRS 9 (Whittet et al. 1996). Each of these identifications will be discussed in turn.

$\mathbf{H}_{2} \mathbf{O}$ : Objects associated with molecular cloud material show the strong $3.0 \mu \mathrm{m}$ stretching vibration of solid $\mathrm{H}_{2} \mathrm{O}$ ice. The broad profile and peak position of this band is generally characteristic of amorphous $\mathrm{H}_{2} \mathrm{O}$ ice (Hagen et al. 1982). In a few sources, narrow substructure reveals the presence of small amounts of crystalline (cubic or hexagonal) ice (Smith et al. 1989). $\mathrm{KAO}$ and ISO spectra show a broad $6.0 \mu \mathrm{m}$ feature (Fig. 1; Tielens \& 
Allamandola 1987a; Schutte et al. 1996). Detailed comparison with laboratory spectra shows that amorphous $\mathrm{H}_{2} \mathrm{O}$ ice is a dominant contributor to this band but that there are also blue and red excess absorption wings. The blue wing, peaking around $5.8 \mu \mathrm{m}$, suggests the presence of carbonyl bearing molecules and a good fit is obtained with $\mathrm{HCOOH} / \mathrm{H}_{2} \mathrm{O}=3 / 100$ (Schutte et al. 1996). Formaldehyde does not provide a good fit to this wing. The red wing may be the absorption counterpart of the $6.2 \mu \mathrm{m} \mathrm{PAH}$ emission feature. Finally, some spectra show the $\mathrm{H}_{2} \mathrm{O}$ librational band as a weak wing on the strong $10 \mu \mathrm{m}$ silicate band (cf., Fig. 1; Whittet et al. 1996; Cox 1989). This band is somewhat difficult to detect in ground based spectra because of continua problems due to lack of spectral coverage (cf., Tielens 1989).

$\mathrm{CH}_{3} \mathrm{OH}$ : The $\mathrm{CH}$ stretching mode of methanol at $3.54 \mu \mathrm{m}$ has been detected as weak substructure on the wing of the $3 \mu \mathrm{m}$ ice band in a number of protostellar spectra (Grim et al. 1990; Allamandola et al. 1992; Brooke et al. 1996). Weak combination bands at $3.9 \mu \mathrm{m}$ and an overtone band at 2.4 $\mu \mathrm{m}$ of solid- $\mathrm{CH}_{3} \mathrm{OH}$ have been detected in W33A and WL 16 , respectively (Allamandola et al. 1992; Sandford et al. 1993). The C-O stretching and $\mathrm{CH}_{3}$ rocking modes - at 9.7 and $8.9 \mu \mathrm{m}$, respectively - have been detected as weak substructure in the $10 \mu \mathrm{m}$ silicate feature towards GL 2136 (Skinner et al. 1992). The $\mathrm{CH}$ and $\mathrm{OH}$ deformation modes of $\mathrm{CH}_{3} \mathrm{OH}$ occur at $6.8 \mu \mathrm{m}$ and a broad and strong absorption feature is present at that wavelength in many spectra (Tielens \& Allamandola 1987a; Schutte et al. 1996). However, detailed comparisons between the observations and laboratory spectra and abundance considerations (see below) suggest that methanol contributes typically only $30 \%$ to this band (Schutte et al. 1996). The multiplicity of $\mathrm{CH}_{3} \mathrm{OH}$ bands detected in several different sources make the identification of solid $\mathrm{CH}_{3} \mathrm{OH}$ very secure.

However, the $\mathrm{CH}_{3} \mathrm{OH} / \mathrm{H}_{2} \mathrm{O}$ abundance ratio is more controversial. Initially, based upon the strong $6.8 \mu \mathrm{m}$ band, $\mathrm{C}_{3} \mathrm{OH} / \mathrm{H}_{2} \mathrm{O}$ ratio of 0.5 was derived (Tielens et al. 1984; Tielens \& Allamandola 1987a). However, the weakness of other $\mathrm{CH}_{3} \mathrm{OH}$ modes indicates a much lower abundance ratio (0.05; Grim et al. 1990). Now, the $9.7 \mu \mathrm{m} \mathrm{C-O} \mathrm{stretching} \mathrm{mode} \mathrm{in} \mathrm{GL}$ 2136 is consistent with a $\mathrm{CH}_{3} \mathrm{OH} / \mathrm{H}_{2} \mathrm{O}$ abundance ratio of 0.05 , derived from column densities, but its peak position implies a much higher ratio (>0.5; Skinner et al. 1992). This suggest that $\mathrm{H}_{2} \mathrm{O}$ and $\mathrm{CH}_{3} \mathrm{OH}$ ices are not well mixed along this sight-line. The presence of distinct ice components with different compositions can also be inferred from $\mathrm{CO}, \mathrm{CO}_{2}$, and OCS observations (see below).

CO: The solid CO band consists generally of two components: a narrow $(\simeq 0.01 \mu \mathrm{m})$ feature near $4.67 \mu \mathrm{m}$ which generally dominates the observed band and a broader underlying component peaking around $4.68 \mu \mathrm{m}$ (Tielens 

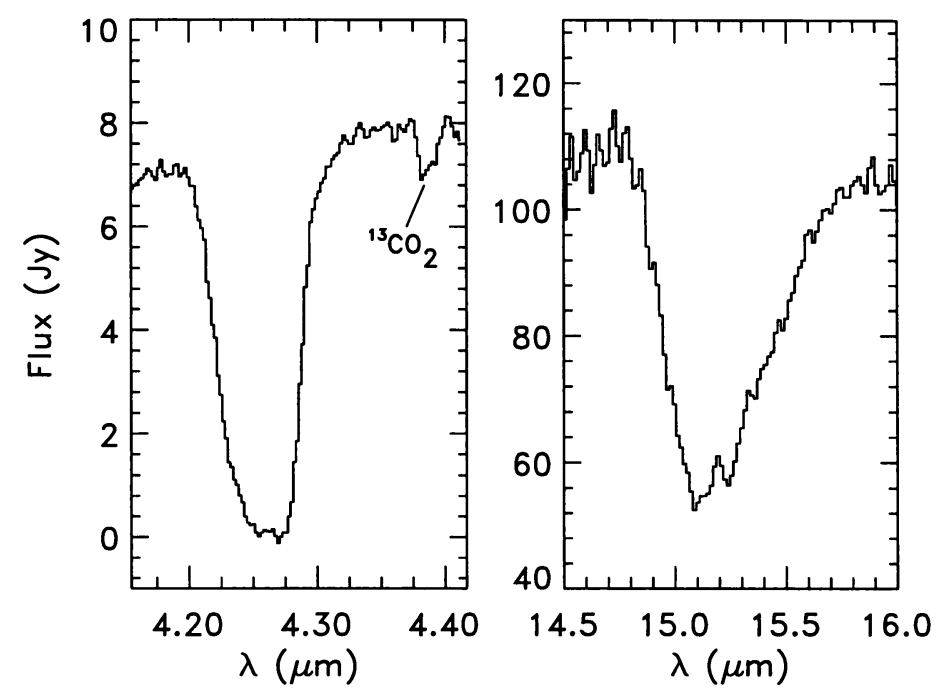

Figure 2. The stretching (left) and bending (right) modes of solid $\mathrm{CO}_{2}$ observed towards NGC 7538 IRS 9 (de Graauw et al. 1996). The resolution of these ISO SWS06 observations has been smoothed to 1000 (left) and 500 (right), respectively. Note the weak ${ }^{13} \mathrm{CO}_{2}$ stretching mode near $4.385 \mu \mathrm{m}$.

et al. 1991; Chiar et al. 1995). The relative strength of these two components as well as the peak position and profile of the narrow component vary from source to source. The broad component can be attributed to $\mathrm{CO}$ mixed in with polar molecules. Because of the predominance of $\mathrm{H}_{2} \mathrm{O}$ ice over other polar species (ie., $\mathrm{CH}_{3} \mathrm{OH}$ ), $\mathrm{CO} / \mathrm{H}_{2} \mathrm{O}$ mixtures with ratios $<10$ are indicated, but this is not proven. The narrow component is characteristic for $\mathrm{CO}$ in an nonpolar environment, consisting for example of $\mathrm{O}_{2}, \mathrm{~N}_{2}$, or $\mathrm{CO}$ itself. The composition of this component varies then from source to source, reflecting the local physical conditions.

$\mathbf{C O}_{2}$ : SWS on ISO has revealed the widespread presence of solid $\mathrm{CO}_{2}$ in molecular clouds (Fig. 2; de Graauw et al. 1996). Both the stretching mode near 4.27 and the bending mode near $15.3 \mu \mathrm{m}$ have been detected in a wide variety of sources. Several sources also show the weaker ${ }^{13} \mathrm{CO}_{2}$ isotope feature at $4.38 \mu \mathrm{m}$ (d'Hendecourt et al. 1996; de Graauw et al. 1996). As for CO, the detailed profiles of these bands indicate the presence of polar and nonpolar $\mathrm{CO}_{2}$ components. Particularly, the $15.3 \mu \mathrm{m}$ band shows substructure which is characteristic for pure $\mathrm{CO}_{2}$ ice.

OCS: A weak feature near $4.9 \mu \mathrm{m}$ was discovered in the spectrum 


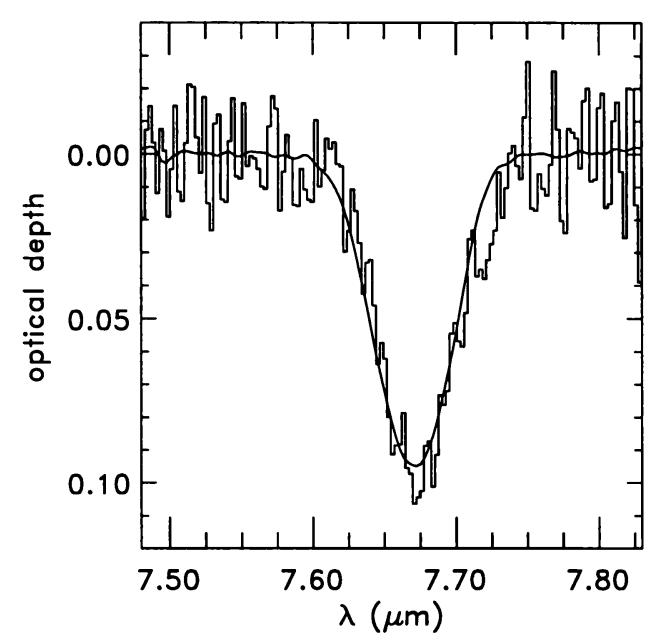

Figure 3. The $7.6 \mu \mathrm{m}$ band observed towards NGC 7538 IRS 9 is compared to the laboratory spectrum of the deformation mode of solid $\mathrm{CH}_{4}$ in an $\mathrm{H}_{2} \mathrm{O}$-rich mixture (Boogert et al. 1996). The resolution of these ISO SWS06 observations is 1500.

of W33A and attributed to solid carbonyl sulphide (Geballe et al. 1985; Palumbo et al. 1995). It has now been observed towards two other sources, GL 989 and Mon R2 IRS 2 (Palumbo et al. 1997). The peak position and width indicate the presence of OCS in a polar environment but the best fit to the data is obtained for traces of OCS in methanol rather than $\mathrm{H}_{2} \mathrm{O}-$ rich mixtures (Palumbo et al. 1995). Yet, the observed column densities of $\mathrm{H}_{2} \mathrm{O}$ and $\mathrm{CH}_{3} \mathrm{OH}$ indicate that methanol is only a trace component towards W33A. As for the $9.7 \mu \mathrm{m} \mathrm{CH} \mathrm{CH}_{3} \mathrm{OH}$ band observed towards GL 2136, this indicates separate $\mathrm{H}_{2} \mathrm{O}$ and $\mathrm{CH}_{3} \mathrm{OH}$ ice components along the line of sight. Other candidates (ie., $\mathrm{CH}_{3} \mathrm{OH}, \mathrm{CN}, \mathrm{CO}_{3}$ ) have been considered for the 4.9 $\mu \mathrm{m}$ band but these do not fit the observed peak position and profile well. Unfortunately, other OCS modes are too weak to be detected and hence confirmation of this identification will be difficult.

$\mathbf{C H}_{4}$ : Ground-based, airborne and space-based observations have revealed a weak $(\tau \simeq 0.1)$ narrow $(\simeq 0.03 \mu \mathrm{m})$ absorption feature near 7.67 $\mu \mathrm{m}$ in the spectra of several luminous protostars (Lacy et al. 1991; Boogert et al. 1996; 1997). This feature is well fitted by traces of $\mathrm{CH}_{4}$ in polar ices (cf. Fig. 3; Boogert et al. 1996). Unlike solid CO, there is no indication for the presence of $\mathrm{CH}_{4}$ in a nonpolar environment.

XCN: The so-called XCN band near $4.62 \mu \mathrm{m}$ was first discovered in a study of the solid CO band towards W33A (Lacy et al. 1984). Comparison 
with laboratory spectra of photolyzed mixtures suggested an origin in an unspecified nitrile or isonitrile compound ${ }^{1}$. This wavelength region is very characteristic of triple bonds and cumulative double bonds. However, a recent laboratory study of astrophysically relevant (iso)nitrile compounds (Bernstein et al. 1997) has not lead to a more specific identification and the XCN nomenclature has kept its ground. Recent studies have shown that the $4.62 \mu \mathrm{m}$ band is present in the spectra of a large number of luminous protostars (Pendleton et al. 1997). A note of caution is appropriate here. As the spectrum of L1551 IRS 5 illustrates (Tegler et al. 1995), gaseous CO lines are easily mistaken for the solid XCN band at resolutions $<1500$.

\section{The composition of interstellar ices}

Table 1 summarizes the observed composition of interstellar ice towards the deeply embedded object NGC 7538 IRS $9 . \mathrm{H}_{2} \mathrm{O}$ dominates the column density of ice by a large factor. The carbon-bearing species, $\mathrm{CO}, \mathrm{CO}_{2}$, and $\mathrm{CH}_{3} \mathrm{OH}$, are less abundant by a factor of 10 to 20 , while $\mathrm{CH}_{4}$ is at the $1 \%$ level compared to $\mathrm{H}_{2} \mathrm{O}$. This dominance of $\mathrm{H}_{2} \mathrm{O}$ is directly evident from the complete IR spectrum of this source (Fig. 1), since the $\mathrm{H}_{2} \mathrm{O}$ absorption features are more conspicuous than any of the other ice features and yet the intrinsic strength of absorption features are very similar for most molecules. An exception should be made for the homonuclear molecules $\mathrm{O}_{2}$ and $\mathrm{N}_{2}$ which have no IR active vibrational modes. While the solid matrix will perturb these modes into (very weak) activity, these bands will be difficult to detect (Ehrenfreund et al. 1992).

Nevertheless, this global ice inventory is somewhat misleading since, as emphasized in $\S 2$, analysis of the observed profiles of $\mathrm{CO}, \mathrm{CO}_{2}, \mathrm{CH}_{3} \mathrm{OH}$, and OCS have revealed the presence of a number of independent ice components along most lines of sight. In particular, $\mathrm{H}_{2} \mathrm{O}$ is mainly located in an $\mathrm{H}_{2} \mathrm{O}$ rich ice, as evident from the width and peak position of the 3 and $6 \mu \mathrm{m}$ bands. Solid CO, on the other hand, is mainly carried by an apolar ice component dominated by $\mathrm{O}_{2}, \mathrm{~N}_{2}$, or $\mathrm{CO}$ itself. Solid $\mathrm{CO}_{2}$ studies also reveal the presence of multiple ice mantle components along many lines of sight. Finally, while not proven for this particular source, $\mathrm{CH}_{3} \mathrm{OH}$ may also be carried by a separate methanol-rich ice component.

The most striking aspect of the IR spectrum of interstellar ices is the apparent simplicity of the composition. Furthermore, the abundance of these species is much higher in the ice than in the gas phase of dark clouds. This preference for saturated species and this abundance difference implicates a chemical origin different from ion-molecule chemistry. Except for the XCN band (at $4.62 \mu \mathrm{m}$ ), which is rather weak, and the $6.85 \mu \mathrm{m}$ band, whose

\footnotetext{
${ }^{1}$ With an apology to chemists, the $\mathrm{X}$ in XCN does not stand for halogen.
} 
TABLE 1. Interstellar ice composition towards NGC 7538 IRS 9

\begin{tabular}{llcc}
\hline Species & $\begin{array}{l}\text { Features } \\
{[\mu \mathrm{m}]}\end{array}$ & Abundance $^{a}$ & $\begin{array}{c}\mathrm{T}_{\text {sub }}^{b} \\
{[\mathrm{~K}]}\end{array}$ \\
\hline $\mathrm{H}_{2} \mathrm{O}$ & $3.08,6.0,(12)$ & 100 & 90 \\
$\mathrm{CO}$ (polar) & 4.68 & 3 & $90^{c}$ \\
$\mathrm{CO}$ (apolar) & 4.67 & 6 & $17^{d}$ \\
$\mathrm{CH}{ }_{3} \mathrm{OH}$ & $3.5,6.8(2.4,3.9,8.9,9.7)$ & 8 & $85^{d}$ \\
$\mathrm{CO}_{2}$ (polar) & $4.27,15.3$ & 9 & $90^{c}$ \\
$\mathrm{CO}_{2}$ (apolar) & $4.27,15.3$ & 6 & $50^{d}$ \\
$\mathrm{CH}_{4}$ & 7.6 & 2 & $90^{c}$ \\
$\mathrm{XCN}$ & 4.62 & $(4)^{e}$ & $?$ \\
$\mathrm{OCS}$ & 4.9 & $0.1^{f}$ & $85^{g}$ \\
\hline
\end{tabular}

${ }^{a}$ Relative to $\mathrm{H}_{2} \mathrm{O}=100$.

${ }^{b}$ Sublimation temperature.

${ }^{c}$ Trace species can be trapped until $\mathrm{H}_{2} \mathrm{O}$ evaporation at $90 \mathrm{~K}$.

${ }^{d}$ Pure solid.

${ }^{e}$ Calculated assuming typical nitrile intrinsic strength.

$f$ The reality of the OCS band in the SWS spectrum of this source has to be confirmed.

$g$ Assuming that OCS is a trace species in $\mathrm{CH}_{3} \mathrm{OH}$ (see text).

identification is somewhat controversial, no unidentified bands are present. Clearly, grain (surface) chemistry heavily favors simple species and, as a corrolary, the role of UV photolysis seems limited. Finally, the low abundance of $\mathrm{CH}_{4}$ and $\mathrm{NH}_{3}(<5)$ relative to $\mathrm{H}_{2} \mathrm{O}$ suggest that the accreting gas was largely molecular in composition (eg., low $\mathrm{C} / \mathrm{CO}$ and $\mathrm{N} / \mathrm{N}_{2}$ ratios).

\section{Grain surface chemistry}

Under typical conditions, a grain accretes a gas phase species at a rate of about 1 species a day. Laboratory experiments show that, at $10 \mathrm{~K}$, the migration timescales of the atoms $\mathrm{H}(\mathrm{D}), \mathrm{C}, \mathrm{N}$, and $\mathrm{O}$ are short compared to this accretion timescale, while heavier atoms, radicals, and molecules are immobile. Thus, a migrating species can scan the whole surface for a coreactant and react before another species is accreted (Tielens \& Hagen 1982). As a result, grain surface chemistry is diffusion limited (ie., limited by the rate at which species are transported to the surface) rather than reaction limited (ie., limited by the coreactant collision rate on the grain surface). This point was well appreciated by early studies on grain surface chemistry (c.f., Hollenbach \& Salpeter 1971; Allen \& Robinson 1977; Tielens \& Hagen 1982; Tielens \& Allamandola 1987b). In recent years, however, calculations have been performed in the reaction limited regime (cf., Hasegawa et al. 
1992; Shalabiea \& Greenberg 1994; Bergin et al. 1995; d'Hendecourt et al. 1985). This may lead to erroneous results (Charnley et al. 1996) and the effects of this have not been completely assessed.

Thus, grain surface chemistry is limited to reactions among these migrating radicals and between these migrating radicals and immobile species (Tielens \& Hagen 1982; Tielens \& Allamandola 1987b). Since much of the $\mathrm{C}$ is locked up in $\mathrm{CO}$ in the accreting gas, by necessity, the grain surface chemistry reaction network is rather limited and will favor simple, saturated species over carbon chains. Besides radical-radical reactions, reactions between radicals and unsaturated species with even numbers of electrons, such as $\mathrm{CO}$, should also be considered if the activation barrier is small. Hydrogenation reactions can be particularly important since atomic $\mathrm{H}$ rapidly tunnels through an activation barrier as large as $2000 \mathrm{~K}$ on a day's time scale (i.e., accretion timescale for a coreactant; Tielens \& Hagen 1982). Based upon this, the following hydrogenation route of $\mathrm{CO}$ has been proposed as the major source of $\mathrm{CH}_{3} \mathrm{OH}$ on grain surfaces (Tielens \& Allamandola 1987b; Charnley et al. 1996),

$$
\mathrm{CO} \stackrel{\mathrm{H}}{\longrightarrow} \mathrm{HCO} \stackrel{\mathrm{H}}{\longrightarrow} \mathrm{H}_{2} \mathrm{CO} \stackrel{\mathrm{H}}{\longrightarrow} \mathrm{CH}_{3} \mathrm{O} \stackrel{\mathrm{H}}{\longrightarrow} \mathrm{CH}_{3} \mathrm{OH}
$$

The first step in this scheme, hydrogenation of $\mathrm{CO}$, occurs at low temperatures $(10-30 \mathrm{~K})$ in icy matrices despite the $1000 \mathrm{~K}$ activation barrier (van IJzendoorn et al. 1984). The hydrogenation steps involving the radicals $\mathrm{HCO}$ and $\mathrm{CH}_{3} \mathrm{O}$ are likely to occur on "collision" on grain surfaces. However, hydrogenation of $\mathrm{H}_{2} \mathrm{CO}$ is not well established. In the gas phase, atomic $\mathrm{H}$ abstracts an $\mathrm{H}$ from $\mathrm{H}_{2} \mathrm{CO}$ to reform $\mathrm{HCO}$ with an activation barrier of $1850 \mathrm{~K}$. Experiments yield a very uncertain estimate for the activation barrier for adduct formation $(\simeq 2000 \pm 2000 \mathrm{~K}$; Westbrook \& Dryer 1979).

Reactions of $\mathrm{H}$ with $\mathrm{CO}$ and its progeny have also been studied experimentally by Hiraoke et al. (1994). In these experiments, a hydrogen discharge beam was sprayed over a $\mathrm{CO}$ film at $10 \mathrm{~K}$. During warm-up, the abundance ratio of $\mathrm{CO}: \mathrm{H}_{2} \mathrm{CO}: \mathrm{CH}_{3} \mathrm{OH}$ was measured by mass spectrometry to be $1: 6 \times 10^{-4}: 10^{-4}$, validating the chemical scheme of Eq. (1). Moreover, from the observed abundance ratios, it follows that hydrogenation of $\mathrm{H}_{2} \mathrm{CO}$ is favored over hydrogenation of $\mathrm{CO}$ by a factor $\simeq 10^{3}$. Now, in these experiments, the overall efficiency of the conversion is very low despite the very high $\mathrm{H}$-flux $\left(10^{5}\right.$ per site per s), leading to speculations that $\mathrm{H}$-abstraction from $\mathrm{H}_{2} \mathrm{CO}$ dominates over $\mathrm{H}$-addition (Hiraoka et al. 1994). Actually, while that channel in principle might compete, it is easy to show that under the laboratory conditions, the $\mathrm{H}$-surface coverage is so high that most of the $\mathrm{H}$ will react with another $\mathrm{H}$ forming $\mathrm{H}_{2}$, which thermally evaporates, before $\mathrm{H}$ can tunnel through the activation barrier 


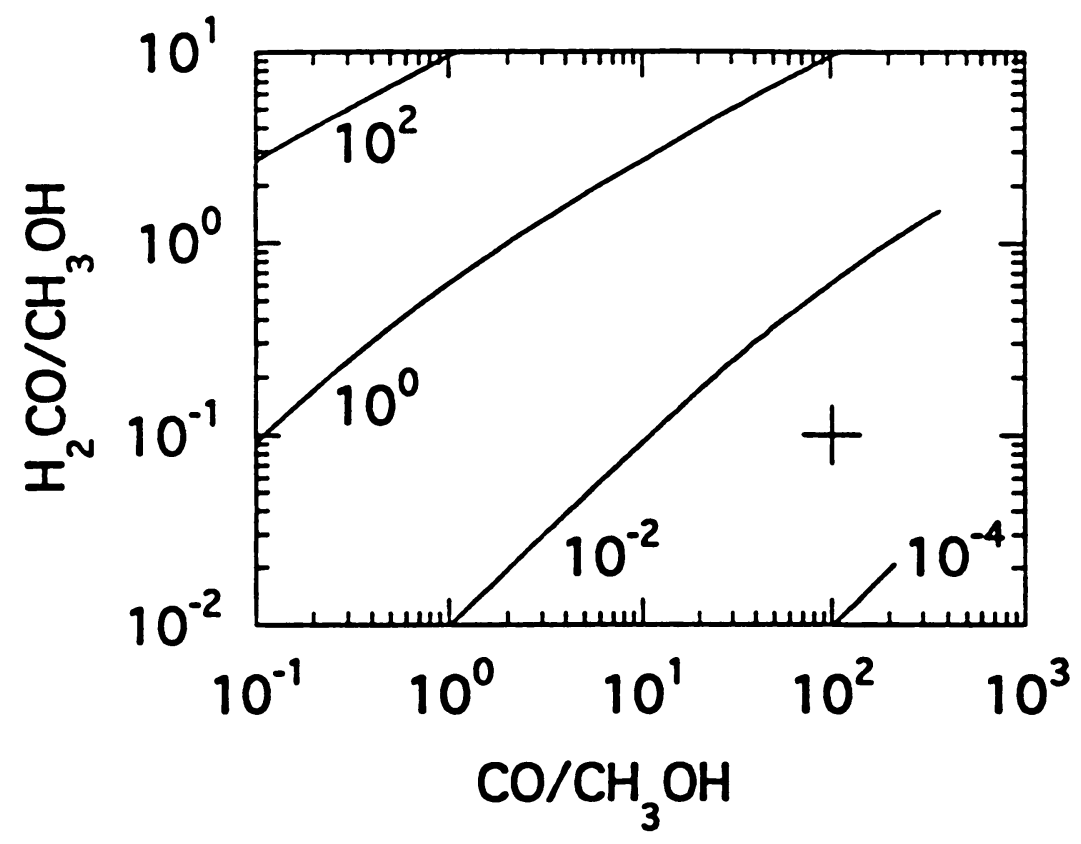

Figure 4. The abundance ratio of $\mathrm{H}_{2} \mathrm{CO}$ and $\mathrm{CO}$ relative to $\mathrm{CH}_{3} \mathrm{OH}$ calculated following equation 1 . The curves show results for different $\phi$ 's - the ratio of the probability for $\mathrm{H}$ to react with $\mathrm{CO}$ relative to $\mathrm{H}_{2} \mathrm{CO}$. Along each curve the $\mathrm{H} / \mathrm{CO}$ accretion probability varies from small (left) to large (right). The cross indicates observed abundances in the Orion Compact Ridge.

associated with the CO reaction (cf., Tielens \& Hagen 1982). In interstellar space, the hydrogen flux is so low $\left(10^{-10}\right.$ per site per $\left.\mathrm{s}\right)$ that an accreted $\mathrm{H}$ atom always tunnels through the $\mathrm{CO}$ barrier before the next $\mathrm{H}$ atom arrives; i.e., this system is in the reaction limit in the lab, but the diffusion limit prevails in the ISM.

Adopting this chemical scheme, activation barriers for these reactions can be derived which are consistent with interstellar observations (Charnley et al. 1996). In this scheme, the relative abundance of $\mathrm{CO}, \mathrm{H}_{2} \mathrm{CO}$ and $\mathrm{CH}_{3} \mathrm{OH}$ will depend on the relative accretion rates of $\mathrm{H}$ and $\mathrm{CO}, p$, and the probability, $\phi$, for a single $\mathrm{H}$ on a grain surface to react with a single $\mathrm{CO}$ relative to the probability that an $\mathrm{H}$ will react with a single $\mathrm{H}_{2} \mathrm{CO}$; i.e., the competition between $\mathrm{CO}$ and $\mathrm{H}_{2} \mathrm{CO}$ for atomic $\mathrm{H}$. Figure 4 shows the abundance ratios of $\mathrm{CO} / \mathrm{CH}_{3} \mathrm{OH}$ and $\mathrm{H}_{2} \mathrm{CO} / \mathrm{CH}_{3} \mathrm{OH}$ for different reaction probability ratios. For each $\phi$, the $\mathrm{H}_{2} \mathrm{CO} / \mathrm{CH}_{3} \mathrm{OH}$ and $\mathrm{CO} / \mathrm{CH}_{3} \mathrm{OH}$ ratios increase as $p$ decreases, as there is less $\mathrm{H}$ around to hydrogenate. Since solid state $\mathrm{CO}$ abundances are heavily influenced by outgassing (Tielens et al. 1991), IR observations of icy grain mantles cannot directly be used to 
constrain the parameters in this model. However, the gas phase composition of molecular cloud hot cores is thought to reflect the outgassing of ice grain mantles by nearby luminous protostars and hence can be used to test these models. The cross in Figure 4 indicates the values of the $\mathrm{H}_{2} \mathrm{CO} / \mathrm{CH}_{3} \mathrm{OH}$ and $\mathrm{CO} / \mathrm{CH}_{3} \mathrm{OH}$ ratios observed in the gas phase in the Orion compact ridge (Irvine et al. 1987). Comparing to the models, we derive values of 0.02 and $10^{-3}$ for $p$ and $\phi$, respectively. The latter is in good agreement with the experiments cited above. Thus, within the context of this model, the conditions which eventually lead to the compact ridge composition were accretion during an atomic- $\mathrm{H}$-poor phase (ie., high gas density) and a low activation barrier for the formation of $\mathrm{CH}_{3} \mathrm{OH}$ from $\mathrm{H}_{2} \mathrm{CO}$.

Besides hydrogen-reactions, the long accretion timescale may also allow oxidation reactions to occur. However, oxygen will have to overcome any activation barrier through thermal hopping and as a result the limiting activation energy is much lower, $\simeq 400 \mathrm{~K}$. Nevertheless, this might still be sufficient to allow oxidation of $\mathrm{CO}$ and this may be the predominant grain surface route towards $\mathrm{CO}_{2}$ (Tielens \& Hagen 1982).

\section{The origin of multiple ice components}

Two general classes of models have been proposed to account for the presence of more than one independent ice mantle component along most lines of sight, based upon chemistry and based upon outgassing (Tielens et al. 1991). Likely, both are of importance in interstellar molecular clouds.

Chemically driven diversity: The chemical composition of the accreting gas may vary along the line of sight. Of particular importance here is the atomic $\mathrm{H}$ over $\mathrm{CO}$ ratio. Since CO locks up most of the elemental C, the $\mathrm{CO}$ density increases linearly with density. In contrast, cosmic ray ionization provides a constant atomic $\mathrm{H}$ density, independent of the total density. Hence, regions with low density will have a high, gas phase, $\mathrm{H} / \mathrm{CO}$ ratio and hydrogenation will lead to a polar grain mantle. High density regions, however, will have a low $\mathrm{H} / \mathrm{CO}$ ratio and grain mantles will mainly consist of inert species such as $\mathrm{CO}, \mathrm{O}_{2}$, and $\mathrm{CO}_{2}$ (Tielens \& Hagen 1982). This is illustrated in Figure 5 (Charnley \& Tielens 1997). The reaction network for this calculation includes the hydrogenation and oxidation reactions of $\mathrm{CO}$ and $\mathrm{O}_{2}$. At low densities, $\mathrm{H}_{2} \mathrm{O}$ and $\mathrm{CH}_{3} \mathrm{OH}$ dominate the ice mantle composition while, at high density, $\mathrm{CO}$ and $\mathrm{O}_{2}$ predominate. There is also a (small) intermediate density regime in which actually $\mathrm{CH}_{3} \mathrm{OH}$ is more abundant than $\mathrm{H}_{2} \mathrm{O}$, because the activation barrier for $\mathrm{H}$ reaction with $\mathrm{O}_{2}$ is slightly higher than for reaction with CO (Tielens \& Hagen 1982). Note that in this scheme, $\mathrm{H}_{2} \mathrm{O}$ is mainly formed from $\mathrm{O}_{2}$ through the peroxide, $\mathrm{H}_{2} \mathrm{O}_{2}$ rather than by direct hydrogenation of atomic O. Finally, the solid 

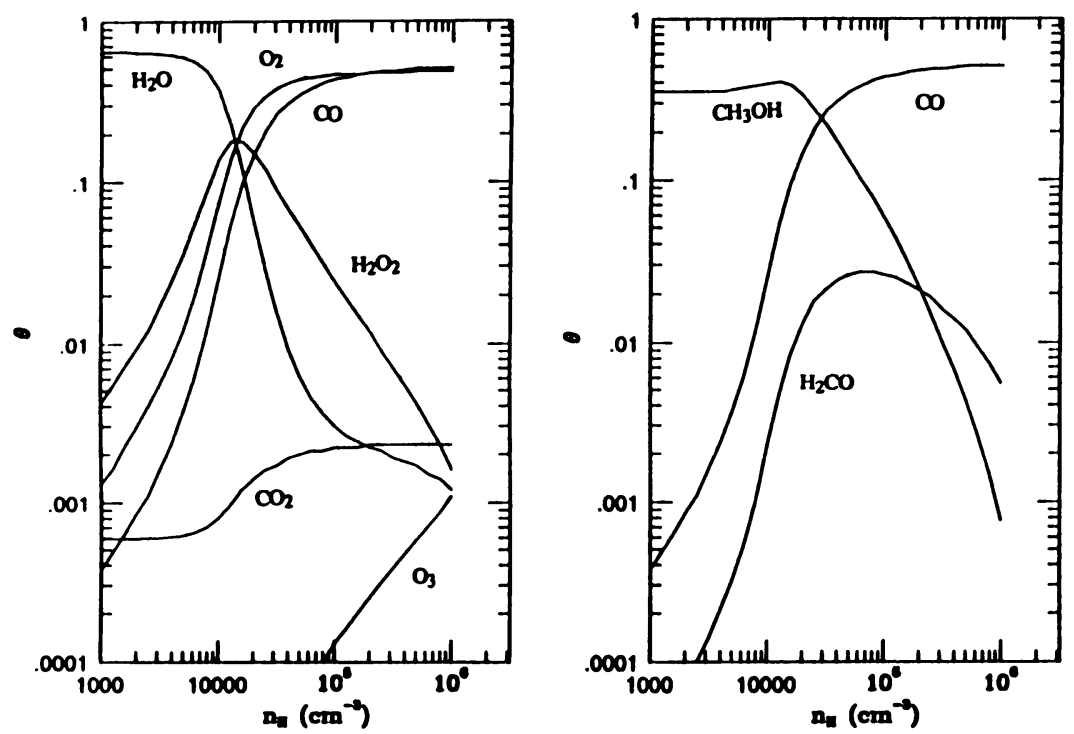

Figure 5. Grain mantle abundances calculated using a surface chemistry scheme which includes hydrogenation and oxidation of $\mathrm{CO}$ and $\mathrm{O}_{2}$ (Charnley \& Tielens 1997).

$\mathrm{CO}_{2}$ abundance is always quite low in this particular calculation, reflecting the low atomic $\mathrm{O}$ over molecular $\mathrm{O}_{2}$ ratio adopted for the accreted gas. A higher $\mathrm{CO}_{2}$ abundance would result if this ratio were assumed to be higher (Charnley \& Tielens 1997). While the gas phase $\mathrm{O} / \mathrm{O}_{2}$ ratio is presently very uncertain, SWAS may remedy that.

Within this class of models, hydrogenated species are preferentially located in grain mantles accreted at low densities where the atomic $\mathrm{H}$ accretion rate is relatively high. Inert or oxidized (if $\mathrm{O} / \mathrm{O}_{2}$ is high) grain mantles reflect then higher density conditions along the line of sight.

Volatility driven diversity: Different species have different volatilities and hence warm up may lead to the preferential loss of some species and an enrichment of others. Sublimation temperatures of a variety of (mixed) molecular ices have been measured in the lab (Sandford \& Allamandola $1990 ; 1993$; Kouchi 1990) and recently reviewed by Tielens \& Charnley (1996; c.f., Table 1).

Sublimation is of particular interest for regions around protostars. Schematically, the observations can then be reconciled with the warm-up of apolar grain mantles, accreted in the pre-protostar dark cloud phase, with a composition of, say, $\mathrm{CO}: \mathrm{CO}_{2}: \mathrm{CH}_{3} \mathrm{OH}: \mathrm{H}_{2} \mathrm{O}=100: 10: 0.1: 1$. Warm up of such grain mantles to above $17 \mathrm{~K}$ leads to loss of $\mathrm{CO}$; ie., a grain mantle with composition CO: $\mathrm{CO}_{2}: \mathrm{CH}_{3} \mathrm{OH}: \mathrm{H}_{2} \mathrm{O}=5: 100: 1: 10$ (some of the 
$\mathrm{CO}$ can remain trapped in the ice matrix). Even closer to the star, $\mathrm{CO}_{2}$ is driven off and the grain mantle composition is then CO: $\mathrm{CO}_{2}: \mathrm{CH}_{3} \mathrm{OH}: \mathrm{H}_{2} \mathrm{O}$ $=5: 5: 10: 100$. Around $80 \mathrm{~K}$, amorphous $\mathrm{H}_{2} \mathrm{O}$ ice crystallizes and $\mathrm{CH}_{3} \mathrm{OH}$ segregates into a separate phase (but still on the same grain). Some trace species can stay behind trapped in a clathrate structure. At $85 \mathrm{~K}, \mathrm{CH}_{3} \mathrm{OH}-$ ice evaporates. Above $90 \mathrm{~K}$, all ices have evaporated and a Hot Core region ensues.

Clearly, a volatility-driven model could explain the general characteristics of multiple grain mantle components along sight-lines towards protostars, but no detailed models have yet been made. These models may also be able to explain the presence of multiple ice mantle components in regions which are not heated by protostars (ie., towards background stars). In particular, heating by (iron) cosmic rays can lead to loss of ice mantle molecules (Léger et al. 1985). Again, more volatile species will be lost preferentially. This heating process may also drive the recombination of stored radicals - produced by FUV irradiation - and the reaction heat released can assist the outgassing process (Schutte \& Greenberg 1991). In either case, ejection will be largely limited to the most volatile molecules; ie., CO. This process might lead to the formation of layered ice mantle structure with a volatile, recently accreted, apolar mantle on top of older, already outgassed, polar ice mantles. Finally, as another twist on this same process, evaporation can also be driven by temperature excursions due to the absorption of a single FUV photon or the heat of a grain surface reaction (Hong \& Greenberg 1974; Allen \& Robinson 1977). Differences in volatility will then again lead to grain mantle segregation. Very volatile molecules such as $\mathrm{CO}$ will be ejected from all grains smaller than $60 \AA$, while polar molecules such as $\mathrm{H}_{2} \mathrm{O}$ can reside on grains as small as $16 \AA$ (Tielens $\&$ Charnley 1996). Thus, the presence of a grain size distribution in dark clouds can lead to multiple grain mantle component formation where the smallest grains (ie., $\mathrm{PAH}$ molecules) are bare, small grains have an $\mathrm{H}_{2} \mathrm{O}$ ice mantle, and large grains have a more nonpolar ice mantle, all along the same line of sight.

\section{Summary}

IR spectroscopy of objects located in or behind molecular clouds show the ubiquitous presence of interstellar ice mantles, consisting of simple molecules $\left(\mathrm{H}_{2} \mathrm{O}, \mathrm{CH}_{3} \mathrm{OH}, \mathrm{CO}, \mathrm{CO}_{2}, \mathrm{OCS}\right.$, and $\left.\mathrm{CH}_{4}\right)$ formed by grain surface reactions among accreted species. In particular, hydrogenation and oxidation reactions of $\mathrm{CO}$ may play a dominant role in determining the composition of interstellar grain mantles. Presently, an absorption feature at $4.62 \mu \mathrm{m}$, attributed to an unspecified nitrile or isonitrile (XCN), may be 
the only sign that more complex molecules are present as well. Extensive laboratory studies suggest that this might reflect FUV photolysis of simple ices.

Contradicting this apparent simplicity of interstellar ices to some extent, the IR spectra also reveal that, along most sight-lines, multiple, independent, ice mantle components are present. The data contain evidence for $\mathrm{H}_{2} \mathrm{O}$-rich ice mantles, nonpolar CO mantles (dominated by $\mathrm{O}_{2}, \mathrm{~N}_{2}$ or $\mathrm{CO}$ ), $\mathrm{CO}_{2}$-rich ice mantles, and $\mathrm{CH}_{3} \mathrm{OH}$-rich ice mantles. While grain surface chemistry can lead to chemical differentiation along sight-lines, likely these multiple components reflect the importance of outgassing of ices in molecular clouds, heated by nearby protostars or temporarily by grain surface reactions taking place or cosmic ray hits.

The launch of ISO has truly opened up the field of infrared spectroscopy of interstellar dust. In sensitivity, resolution $(\simeq 2000)$, and wavelength coverage $(2.5-40 \mu \mathrm{m})$, the SWS is particularly suited for observations of interstellar ices. Some of these observations have been reported here. Over the coming two years, we can expect that a large number of sources will be observed in SWS01 mode, resulting in complete ice "inventories" (cf., Whittet et al. 1996; d'Hendecourt et al. 1996) for perhaps two dozen (mainly luminous) sources. Many of these sources will also be studied for gas phase absorption lines from Hot Core regions (cf., van Dishoeck et al. 1996). An even larger sample, which includes much fainter sources, will be observed over a more limited wavelength region for, e.g., $\mathrm{CO}_{2}$ and $\mathrm{CH}_{4}$ using SWS06 (cf., de Graauw et al. 1996; Boogert et al. 1996). This wealth of data is bound to lead to a greatly increased understanding of the composition of interstellar ices, including their formation and evolution. ISO stems from the Greek root isos which means similar, equal and survives in such words as isomer and isotopomer. However, it is already clear that nothing in the study of interstellar ices will be the same after ISO is done with it.

\section{Acknowledgements}

We thank the SWS Instrument Dedicated Team for their help in obtaining the spectra. We are particularly grateful to Adwin Boogert and Frank Helmich for the reduction of the ISO data shown. Research on interstellar dust at NASA Ames is funded through the Theory, SOFIA, and IR astronomy programs (RTOPs 399-28-10-08, 263-10-97-03, 188-40-01). DCBW is funded by NASA grants NAGW-3144 and NAGW-4039. 


\section{References}

Allamandola, L.J., Sandford, S.A., Tielens, A.G.G.M., Herbst, T., 1992, ApJ, 399, 134 Allen, M., Robinson, G.W., 1977, ApJ, 212, 396

Bergin, E.A., Langer, W.D., Goldsmith, P.F., 1995, ApJ, 441, 222

Bernstein, M., Sandford, S.A., Allamandola, L.J., 1997, ApJ, submitted

Boogert, A.C.A., et al. 1996, A \& A 315, L377

Boogert, A.C.A., Schutte, W., Helmich, F., Tielens, A.G.G.M., Wooden, D., 1997, A \& $A$, in press

Brooke, T.Y., Sellgren, K., Smith, R.G., 1996, ApJ, 459, 209

Charnley, S.B., Tielens, A.G.G.M., 1997, in preparation

Charnley, S.B., Tielens, A.G.G.M., Rodgers, S.D., 1996, ApJ, submitted

Chiar, J.E., Adamson, A.J., Kerr, T.H., Whittet, D.C.B., 1995, ApJ, 455, 234

Cox, P., 1989, A \& A, 225, L1

de Graauw, Th., et al., 1996, A \& A 315, L345

d'Hendecourt, L.J., Allamandola, L.J., Greenberg, J.M., 1985, A \& A, 152, 130

d'Hendecourt, L.B., et al., 1996, A \& A 315, L365

Ehrenfreund, P., Breukers, R., d'Hendecourt, L.B., Greenberg, J.M., 1992, A \& A 260, 431

Geballe, T.R., Baas, F., Greenberg, J.M., Schutte, W., 1985, A \& A, 146, L6

Greenberg, J.M., 1982, in Comets, ed. L. Wilkening, (U of A Press, Tucson), 131

Grim, R., Baas, F., Geballe, T., Greenberg, J., Schutte, W., 1990, A \& A, 243, 473

Hagen, W., Tielens, A.G.G.M., Greenberg, J.M., 1993, A \& AS, 51, 389.

Hasegawa, T.I., Herbst, E., Leung, C.M., 1992, ApJS, 82, 167

Hiraoka, K., Ohashi, N., Kihara, Y., Yamamoto, K., Sato, T., Yamashita, A., 1994, Chem Phys Letters, 229, 408

Hollenbach, D.J., Salpeter, E.E., 1971, JCP, 53, 79

Hong, S.S., Greenberg J.M., 1974, in Galactic Radio Astronomy, eds. F.J. Kerr and S.C., Simonson, (Reidel, Dordrecht), 155

Irvine, W.M., Goldsmith, P.F., Hjalmarson, A., 1987, in Interstellar Processes, eds. D.J. Hollenbach and H.A. Thronson, Jr., (Reidel, Dordrecht), 561

Kouchi, A., 1990, J Crystal Growth, 99, 1220

Lacy, J.H., et al., 1984, ApJ, 276, 533

Lacy, J.H., et al., 1991, ApJ, 376, 556

Léger, A., Jura, M., Omont, A., 1985, A \& A, 144, 147

Palumbo, M.E., Tielens, A.G.G.M., Tokunaga, A.T., 1995, ApJ, 449, 674

Palumbo, M.E., Tielens, A.G.G.M., Geballe, T.R., 1997, in press

Pendleton, Y., Tielens, A.G.G.M., Tokunaga, A.T., 1997, in preparation

Sandford, S.A., and Allamandola, L.J., 1990, ApJ, 355, 357

Sandford, S.A., and Allamandola, L.J., 1993, ApJ, 417, 815

Sandford, S.A., Allamandola, L.J., Geballe, T.R., 1993, Science, 262, 400

Schutte, W.A., Greenberg, J.M., 1991, A \& A, 244, 190

Schutte, W., et al., 1996, A \& A, 315, L333

Shalabeia, O.M., Greenberg, J.M., 1994, A \& A, 290, 266

Skinner C. J., Tielens, A.G.G.M, Barlow, M.J., Justtanont, K., 1992, ApJL, 399, L79

Smith, R.G., Sellgren, K., Tokunaga, A.T., 1989, ApJ, 344, 413

Tegler, S.C., et al. 1995, ApJ, 439, 279

Tielens, A.G.G.M., 1989, in Interstellar Dust, eds. L.J. Allamandola \& A.G.G.M. Tielens (Kluwer, Dordrecht), 239

Tielens, A.G.G.M., Allamandola, L.J., 1987a, in Physical Processes in Interstellar Clouds, eds. G. Morfill, and M. Scholer, (Reidel, Dordrecht), 333

Tielens, A.G.G.M., Allamandola, L.J., 1987b, in Interstellar Processes, eds. D. Hollenbach, H. Thronson, (Kluwer, Dordrecht), 397

Tielens, A.G.G.M., Allamandola, L.J., Bregman, J., Goebel, J., d'Hendecourt, L.B., Witteborn, F.C., 1984, Ap.J., 287, 697 
Tielens, A.G.G.M., Charnley, S.B., 1996, Origins of Life, in press

Tielens, A.G.G.M., Hagen, W., 1982, A \& A, 114, 245

Tielens, A., Tokunaga, A., Geballe, T., Baas, F., 1991, ApJ, 381, 181

van Dishoeck, E.F. et al. 1996, A\&A, 315, L349

van IJzendoorn, L., Allamandola, L.J., Baas, F., Greenberg, J.M, 1983, JCP, 78, 7019

Walmsley, C.M., 1989, in Interstellar Dust, eds. L.J. Allamandola \& A.G.G.M. Tielens (Kluwer, Dordrecht), 263

Westbrook, C.K. Dreyer, F.L., 1979, Combust. Sci. Techn., 20, 125

Whittet, D.C.B., et al., 1996, A \& A, 315, L357

Whittet, D.C.B. 1993, in Dust and Chemistry in Astronomy, eds. T.J. Millar and D.A. Williams, (Institute of Physics, Bristol), 9

\section{Discussion}

Williams: How do you account for the lack of detection of ammonia in the ices? Tielens: Observationally, solid $\mathrm{NH}_{3}$ is difficult to observe because of nearby strong $\mathrm{H}_{2} \mathrm{O}$ ice absorption. Present upper limits are $\simeq 5 \%$ relative to $\mathrm{H}_{2} \mathrm{O}$, which is comparable to gas phase $\mathrm{NH}_{3} / \mathrm{H}_{2} \mathrm{O}$ abundances in hot cores. Solid $\mathrm{NH}_{3}$ is formed by hydrogenation of atomic $\mathrm{N}$ on grain surfaces. Theoretical models show that under relevant conditions most of the $\mathrm{N}$ is in the form of $\mathrm{N}_{2}$ which is unreactive with respect to $\mathrm{H}$ (unlike $\mathrm{O}_{2}$ ). Solid $\mathrm{N}_{2}$ is extremely difficult to observe. I should emphasize that no abundant $\mathrm{N}$-bearing molecule has been identified in interstellar ices.

Schutte: You state that in order to produce the observed $\mathrm{H}_{2} \mathrm{O}$-dominated ice component you have to be close to the protostar and get rid of the more volatile ice species through outgassing. Yet the $\mathrm{H}_{2} \mathrm{O}$-dominated ice is also observed toward background field stars, which exclusively probe cold $(10 \mathrm{~K})$ ice. How can you explain that in your model?

Tielens: Outgassing driven by warm up by a nearby protostar is just one example of a class of models where the different interstellar ice components observed result from differences in volatility. The warm up could also result from cosmic-ray heating, grain-grain collisions, temporal heating by absorption of a single UV photon by a small grain $(<60 \AA)$, etc. All of these would be very relevant for dark clouds.

Greenberg: How do you account for desorption of molecules from icy matter by cosmic rays if there are no radicals (produced by UV) to cause extra heating?

Tielens: Various processes can return volatile molecules such as $\mathrm{CO}$ to the gas phase, including cosmic-rays, grain-grain collisions etc. Ejection of more polar molecules such as $\mathrm{H}_{2} \mathrm{O}$ may be more problematic, but then, observationally, the evidence for $\mathrm{H}_{2} \mathrm{O}$ ejection from grain mantles isn't strong.

Greenberg:: How do you explain the fact that UV processing is unnecessary to account for, say, $\mathrm{H}_{2} \mathrm{CO}$ if $\mathrm{UV}$ processing rates are orders of magnitude higher than surface reaction rates of production?

Tielens: Let me answer this question in two parts. First within the uncertainties, observations of gaseous $\mathrm{H}_{2} \mathrm{CO}$ in dark clouds are well accounted for by ion-molecule reactions. More $\mathrm{H}_{2} \mathrm{CO}$ is observed in hot cores, presumably from evaporating grain mantles. Second, UV photolysis production of $\mathrm{H}_{2} \mathrm{CO}$ relies on the presence of $\mathrm{CH}_{3} \mathrm{OH}$ in the ice. That, of course, begs the question on the origin of the solid $\mathrm{CH}_{3} \mathrm{OH}$. In my opinion, $\mathrm{CH}_{3} \mathrm{OH}$ is likely formed by hydrogenation from $\mathrm{H}_{2} \mathrm{CO}$. 
Hence, at best, UV photolysis may only reform $\mathrm{H}_{2} \mathrm{CO}$ which was formed differently in the first place (through hydrogenation of $\mathrm{CO}$ ?). More likely, any $\mathrm{H}_{2} \mathrm{CO}$ in the grain mantle was just not fully hydrogenated by surface reactions. Hence the presence of solid $\mathrm{H}_{2} \mathrm{CO}$ (even if it had been observed) cannot be used as an argument for the importance of UV photolysis of interstellar ices. In conclusion, after 25 years of speculation, it is important to critically re-examine the importance of UV photolysis and ISO will allow us to do just that. In that respect, N-bearing molecules might be a more unequivocal signpost of UV photolysis.

Henning: Comment: We detected the $\mathrm{CO}_{2}$ absorption feature in quite a number of deeply embedded sources, using ISOPHOT-S. The data are published in the ISO special issue of A\&A by Gürtler et al. 1996.

Jenniskens: The water frost on interstellar grains is in the high density amorphous form. This material has remarkable properties. For one, this solid material becomes of low viscosity when exposed to electron bombardment and probably also UV light. How does your grain chemistry change when bulk reactions can become possible?

Tielens: I have never seen $\mathrm{H}_{2} \mathrm{O}$ ice turn into a liquid at $10 \mathrm{~K}$ in our laboratory experiments, perhaps because we never exposed it to such extreme conditions as in your experiments.

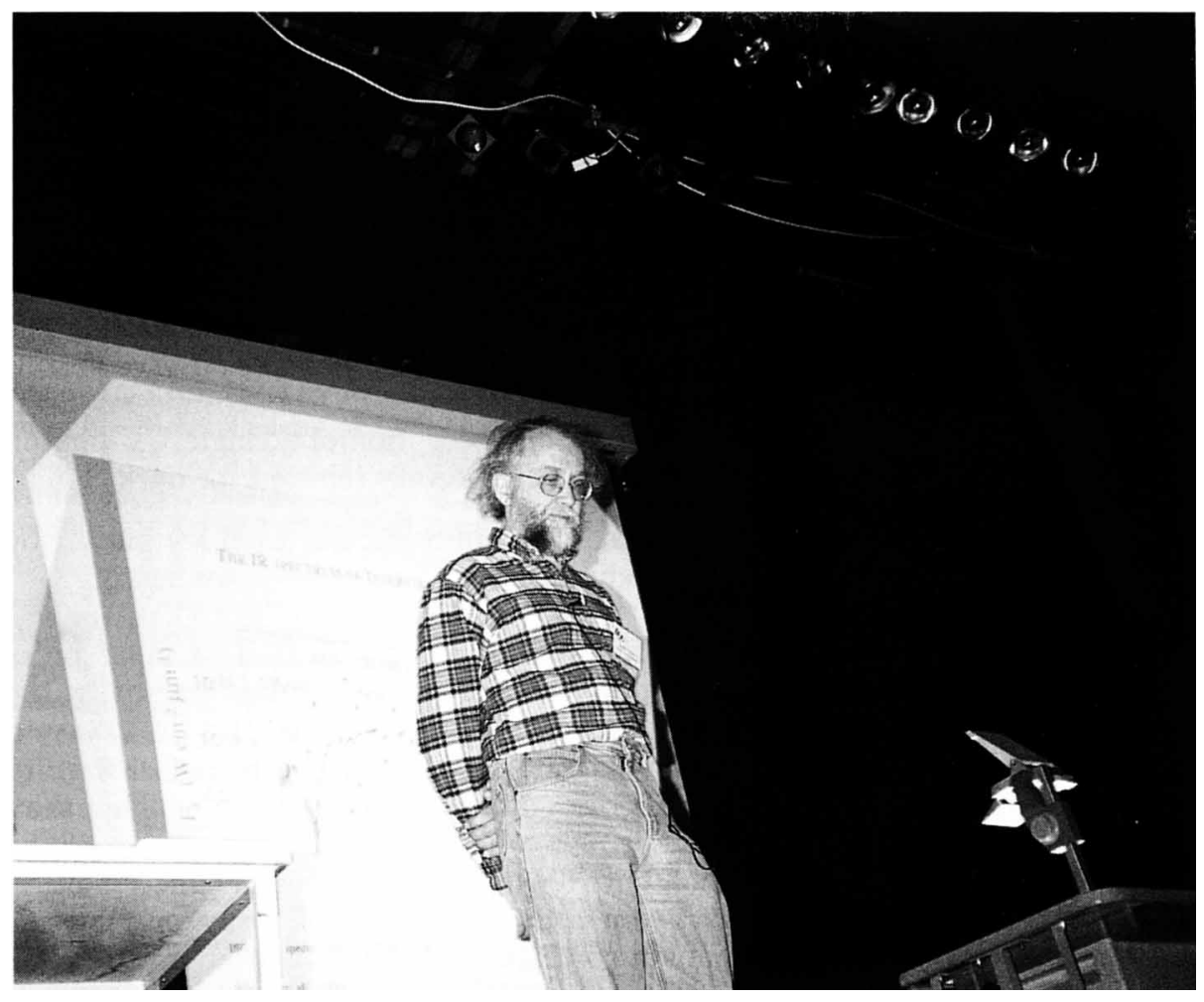

